\title{
-Original-
}

\section{Spontaneous enhancement of synchronous discharges of pyramidal cells and synchronization of recurrent inhibitions in the kindled hippocampus of the rabbit}

\author{
Hajime Harada \\ The Second Department of Physiology (Director: Prof. Yasuichiro Fujita), \\ Nippon Medical School
}

\section{Summary}

1) The rabbit hippocampus was kindled through daily electrical stimulation. The rabbit was then anesthetized with Nembutal, and interictal and brain stem-induced potentials, together with fornix-evoked potentials, were studied by observing the depth profiles of field potentials.

2) Interictal field potentials were characterized by the existence of a large slow positivity when recorded in CA1-CA2 apical dendrite layer. On the other hand, brain stem induced field potential having the shortest latency was recorded invariably as a positive deflection in the apical dendrite layer. These positivities were thought to be the extracellular correlates of the C1-nondependent hyperpolarization of the pyramidal cell, because they were the largest in magnitude in the apical dendrite layer.

3) The largest interictal field potential and supramaximal fornix-evoked field potential were identical with each other in waveform. Both potentials had one or two population spikes at the onset (CA3-CA4) or at the early part (CA1-CA2). This indicated that the synchronous discharges of pyramidal cells were instrumental in generating the large interictal hyperpolarization through synchronous activation of recurrent inhibitory circuits.

Key words: kindled hippocampus, spontaneous synchronous discharges, recurrent inhibition, dendritic positivity, rabbit

\section{Introduction}

Recently, it was shown that the interictal EEG spike in the kindled hippocampus corresponded to the hyperpolarization of the pyramidal cell with or without a preceding single action potentiall). The interictal spike has been therefore referred to as the inhibitory EEG spike2). Since the hyperpolarization in most cases included C1-dependent component, it was obvious that the inhibitory postsynaptic potential (IPSP) constituted at least a part of the hyperpolarization. The hyperpolarization was never preceded by the prolonged depolarization characteristic of the penicillin focus $\left.{ }^{3} \sim 10\right)$. The only excitation which could precede it was a single action potential1,11). Accordingly, it was proposed that while the kindling procedures lowered seizure threshold, they

Present address: The Second Department of Physiology, Nippon Medical School, 1-1-5, Sendagi, Bunkyo-ku, Tokyo, 113 Japan 
facilitated at the same time the synchronous firing of the somatic trigger zones of a number of pyramidal cells, thereby producing powerful inhibition through the synchronous activation of recurrent inhibitory circuits ${ }^{12)}$. It was further disclosed that the inhibitory EEG spike could be elicited by stimulation of the median raphe ${ }^{13}$ ). Both medial raphe-elicited and interictal hyperpolarization had $\mathrm{C} 1$-non-dependent component, in addition to $\mathrm{C} 1$-dependent component. That hyperpolarization was deemed as the hyperpolarization occurring in the dendrites, because it was insensitive to current injection ${ }^{14)}$. The present investigation was undertaken to obtain further evidence for synchronous firing of pyramidal cells during the interictal event and to see if there existed extracellular positivity corresponding to the presumed dendritic hyperpolarization. As will be shown below, positive results were obtained as regards these questions.

\section{Materials and Methods}

(1) Kindling procedures

Eighteen rabbits $(2.0 \sim 3.0 \mathrm{~kg})$ were used. Under pentobarbital sodium anesthesia (Nembutal: $20 \sim 30 \mathrm{mg} / \mathrm{kg}$, i.v. or $50 \sim 65 \mathrm{mg} / \mathrm{kg}$, i.p.), the left hippocampus was implanted with a pair of steel electrodes (100 300 $\mu \mathrm{m}$ in diameter) insulated except at the tips. All rabbits were implanted with a pair of screw electrodes ( $1 \mathrm{~mm}$ in diameter at the base of the screw) in order to monitor the neocortical EEG.

One to two weeks were allowed to pass for each animal to recover from the surgery. Then the daily electrical stimulation was started. The stimulus intensity was $0.1 \sim 3 \mathrm{~mA}$. The left hippocampus was stimulated with a train of $1 \mathrm{~ms}$ pulses at $50 \mathrm{~Hz}$ for $0.5 \sim 1 \mathrm{~s}$. The stimulation was repeated 5 10 times daily with an interval of 5 10 min and lasted for 1 3 weeks. EEGs were monitored with a polygraph (Nihon Kohden RM-80, T.C.: $0.03 \mathrm{~s}$ ).

(2) Acute experiments

In acute experiments the rabbits were anesthetized with Nembutal (20 55 mg/kg, i.v.), immobilized with D-tubocurarine (1 2 mg/kg, i.m.) and maintained on artificial respiration. The animal was fixed in the stereotaxic apparatus (Todai-Noken type). The right hippocampus was examined with microelectrodes by observing extracellular potentials. The tips of microelectrode were artificially broken in order to reduce the resistance. The hippocampus was not exposed, because if exposed, it often lost its normal EEG activity. All the results were obtained in simultaneous extracellular recordings from two different sites in the right hippocampus. Two preamplifiers (WPI M701, M707A) were employed together with a dual beam cathode ray oscillograph (Nihon Kohden VC-9).

In order to monitor EEGs, a concentric electrode ( $800 \mu \mathrm{m}$ in diameter) was introduced into the left hippocampus. For stimulation, another concentric electrode ( $500 \mu \mathrm{m}$ in diameter) was placed on the fornix stereotaxically. The fornix was stimulated with a single pulse of $1 \mathrm{~ms}$ in duration. The brain stem was stimulated through a concentric electrode ( $300 \mu \mathrm{m}$ in diameter) with a train of $200 \mu \mathrm{s}$ pulses at $500 \mathrm{~Hz}$ for $40 \mathrm{~ms}$. Supramaximal stimulus strength was used for fornix and brain stem stimulation. For stimulation, an electronic stimulator (Nihon Kohden SEN-3201) together with an isolating unit was employed.

All the records were obtained from the apical dendrite layer and the pyramidal cell layer in CA1-CA2 region, and CA3-CA4 region by observing the depth profiles of field potentials ${ }^{15 \sim 17}$. 
After the experiments, the location of the stimulating electrodes was examined. The electrode tip on the fornix was directly observed by removing the tissue around it. A direct current $(2 \sim 4 \mathrm{~mA}$, for $2 \sim 5 \mathrm{~s}$ ) was passed through the electrode to make a burnt spot in the brain stem reticular formation. The location of the electrode tip in the brain stem was examined on histological section of $50 \mu \mathrm{m}$ thickness, stained with Nissl. The electrode was located in the area including raphe nuclei ${ }^{2,18 \sim 23)}$.

\section{Results}

The kindled hippocampus was characterized by the presence of large spontaneous (interictal) field potentials (often in excess of $10 \mathrm{mV}$, as recorded with the microelectrode), which corresponded to interictal EEG spikes ${ }^{1}$. In the layer of CA1-CA2 pyramidal cell bodies as well as in CA3-CA4, interictal field potentials and fornix-evoked potentials were identical in that all of them exhibited slow positivity with or without superposed population spikes (Fig. 1, 2, lower traces). In contrast,
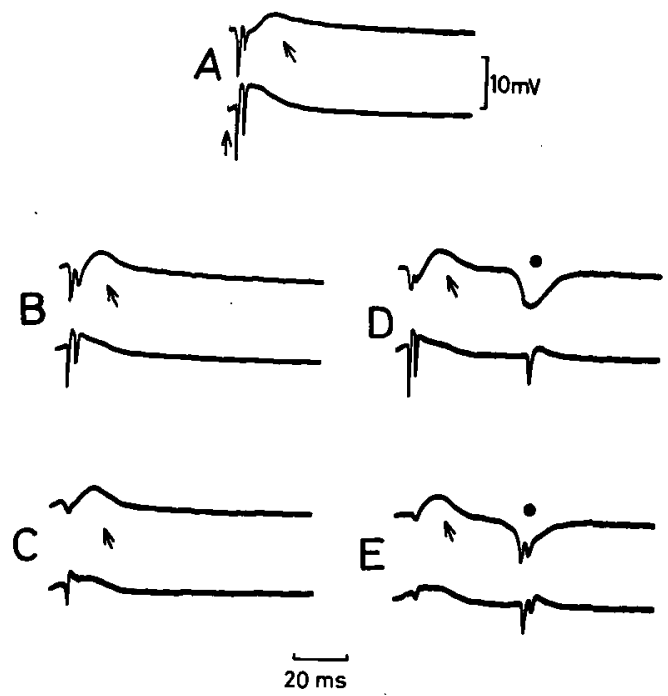

Fig. 1 In this and the following figures: all records were extracellularly taken from the kindled hippocampus; in all records upward deflections indicate positivities at the focal electrode; upper and lower traces were simultaneously recorded. Upper traces: obtained from the apical dendrite layer in CA1-CA2. Lower traces: potentials from the pyramidal cell layer in CA1-CA2. A: evoked potential occurring in response to supramaximal stimulation of the fornix (upward arrow). B-E: potentials occurring spontaneously. Both spontaneous and stimulus-produced potentials consist of population spikes followed by a slow positivity (A, B.E, the potentials appearing immediately after the onset of the sweep). Spontaneous potentials had a larger slow positivity than that produced by fornical stimulation (oblique arrows). Dots indicate spontaneous potentials without the slow positivity $(\mathrm{D}, \mathrm{E})$.

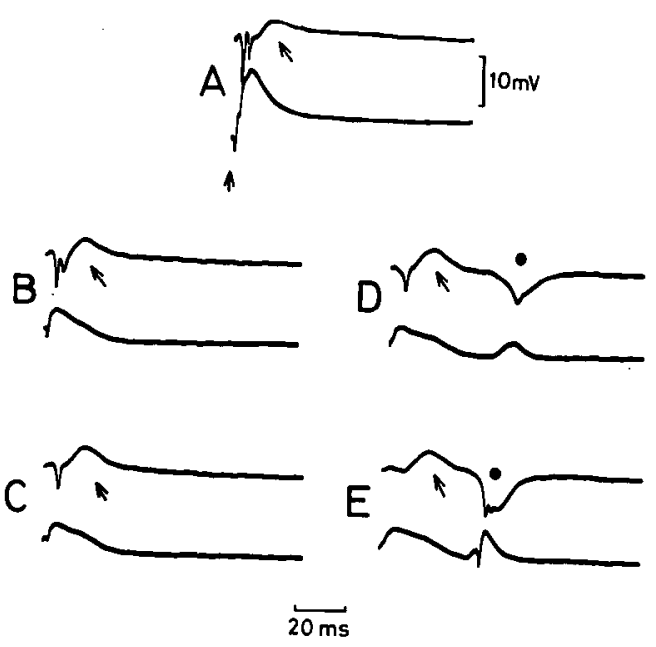

Fig. 2 Upper traces: recorded from the apical dendrite layer in CA1-CA2. Lower traces: obtained from CA3-CA4. A: supramaximal fornix-evoked potential (upward arrow). B-E: potentials occurring spontaneously. Spontaneous potentials resemble the fornix-evoked potential in waveform except that the former has a large slow positivity than that of the latter (oblique arrows). Dots indicate spontaneous potentials which show no slow positivity (D, E). 
in the layer of CA1-CA2 apical dendrite layer, two types of interictal field potentials were distinguished. Namely, the supramaximal fornix-evoked potential consisted of a slow negativity with superposed population spikes followed by a slow positivity (Fig. 1A, Fig. 2A, upper traces). The interictal field potentials in Fig. 1B and Fig. 2B (upper traces) were essentially identical in waveform with the supramaximal fornix-evoked potential (Fig. 1A, Fig. 2A, upper traces). Nevertheless, the slow positivity was obviously enhanced in the interictal field potentials. The interictal field potentials in Fig. 1C, E and Fig. 2C, D (arrows in upper traces) exhibited much less negativity as compared with the supramaximal fornix-evoked potential, while their slow positivity remained enhanced. The interictal field potential in Fig. 2E (arrow in upper trace) showed only the vestige of the slow negativity, in effect appearing as almost a pure positivity. On the other hand, the interictal field potentials in Fig. 1D, E, Fig. 2D, E (dots in upper traces) consisted of pure negativity. Thus, interictal field potentials in the apical dendrite layer were basically categorized into two types: the one exhibiting enhanced slow positivity and the other showing only slow negativity. On the other hand, the interictal field potentials in the layer of CA1-CA2 pyramidal cell

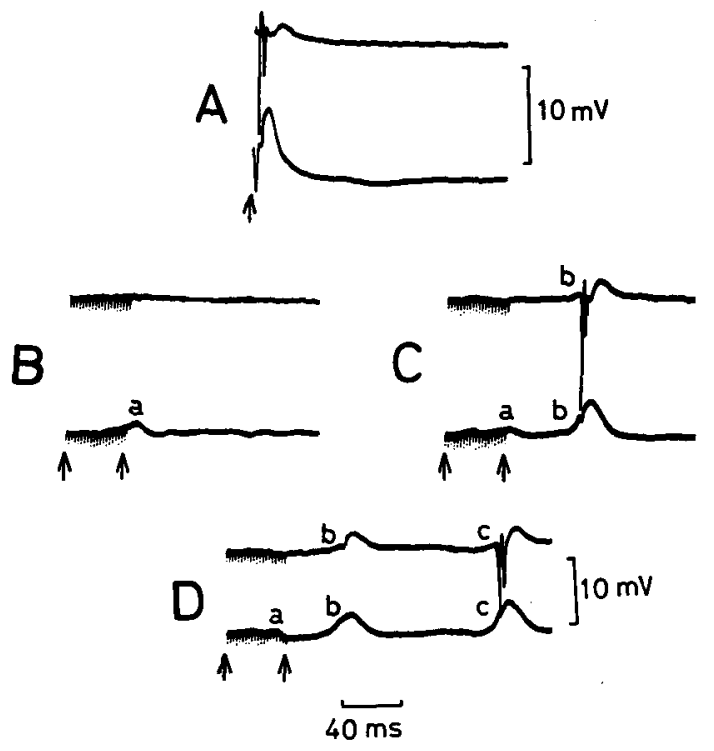

Fig. 3 Upper traces: obtained from the pyramidal cell layer in CA1-CA2. Lower traces: recorded from CA3-CA4. A: supramaximal fornix-evoked potential (upward arrow). B-D: potentials produced by supramaximal brain stem stimulation $(500 \mathrm{~Hz}$ for $40 \mathrm{~ms})$. Brain stem stimulation induced a series of potential changes. Potential $a$ was not discernible in the pyramidal cell layer in CA1-CA2 (B-D). Potential $c$ was not always induced (C, D). Potential $b, c$ produced by brain stem stimulation and fornix-evoked potential are essentially identical in waveform which consists of population spikes followed by a slow positivity (A, C-b, D-c), provided brain stem-induced potentials were the largest.

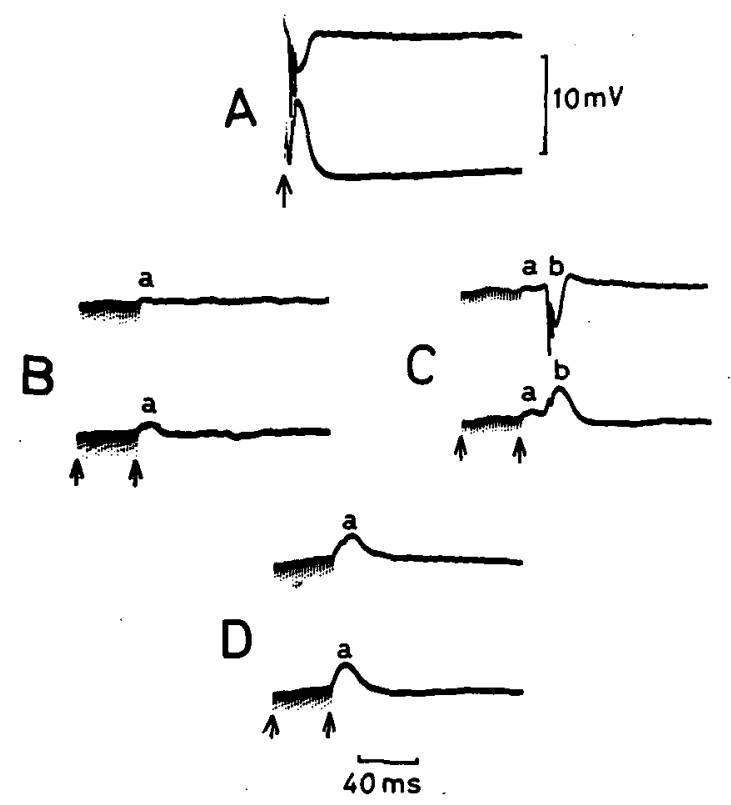

Fig. 4 Upper traces: recorded from the apical dendrite layer in CA1-CA2. Lower traces: recorded from CA3-CA4. A: supramaximal fornix-evoked potential (upward arrow). B-D: potentials produced by supramaximal stimulation of the brain stem $(500$ $\mathrm{Hz}$ for $40 \mathrm{~ms}$ ). Potential $a$ was observed as a positivity in both CA1-CA2 and CA3-CA4. When potential $b$ was the largest, it mimicked the fornix-evoked potential (c-b). 
bodies and CA3-CA4 all exhibited slow positivity, regardless of which of the two types occurred in the apical dendrite layer (Fig. 1, 2).

It is reported that $500 \mathrm{~Hz}$ stimulation of the brain stem elicited three EEG spikes whose intracellular correlates were hyperpolarizations of the pyramidal cell (inhibitory EEG spikes)2). They were referred to as EEG spikes $a, b, c$ and hyperpolarizations $a, b, c$, respectively, according to their latencies. In the present experiments, three groups of field potentials corresponding in latency to EEG spikes $a, b, c$ were observed. Field potential $a$ (latency: $30 \sim 40 \mathrm{~ms}$, as measured from the onset of the stimulation) was hardly discernible in the layer of CA1-CA2 pyramidal cell bodies (Fig. 3B D, upper traces), but could be observed in the CA1-CA2 apical dendrite layer (Fig. 4B D, upper traces). It was in CA3-CA4 that field potential $a$ was most conspicuously observed (Fig. 3, 4, lower traces in B D). Occasionally it was enhanced not only in CA3-CA4, but also in CA1-CA2 apical dendrite layer (Fig. 4D). On the other hand, field potentials $b$ (latency: $55 \sim 104 \mathrm{~ms}$ ) and $c$ $(130 \sim 200 \mathrm{~ms})$ were seen in all the layers studied (Fig. 3, 4). Field potential $a$ occurred most consistently, as observed in CA3-CA4 (incidence: 27 in 30 successive stimulation); field potential $b$ less consistently (incidence: 23 ) and field potential $c$ least consistently (incidence: 7 ). Similarly as in the case of inhibitory EEG spikes $b$, $c$, whenever field potential $b$ was small, field potential $c$ appeared (Fig. 3D). Comparison of Fig. 3C and D makes it evident that field potentials $b$ and $c$ are similar in waveform. Both of them were at their largest similar also to the supramaximal fornix-evoked potential (Fig. 3A, C, D). Both field potentials $a$ and $b$ exhibited slow positivity in CA3-CA4 (Fig. $\mathbf{3}, \mathbf{4}$, lower traces in $\mathrm{B} \sim \mathrm{D}$ ), but in CA1-CA2 apical dendrite layer the former invariably showed slow positivity whereas the latter always exhibited slow negativity (Fig. 4B D, upper traces).

\section{Discussion}

The present experiments revealed the presence of positivity in CA1-CA2 apical dendrite layer during interictal and barin stem elicited field potentials. The positivity could be either the hyperpolarization occurring in the dendrites or the current source to the depolarization taking place in the cell body. The latter possibility can be gainsaid on the ground that in the layer of CA1-CA2 pyramidal cell bodies either no slow potential changes were observed corresponding to brain stemelicited field potential $a$, or slow positivity of smaller magnitude occurred corresponding to interictal dendritic positivity. It can be therefore concluded that the dendritic positivity denotes the presence of hyperpolarization in the dendrites. That slow positivity could occur in the dendrites and the soma simultaneously could be explained by the simultaneous occurrence of hyperpolarization in both portions of the pyramidal cell. In fact in most cases $\mathrm{C} 1$-dependent hyperpolarization occurred simultaneously with $\mathrm{C} 1$-non-dependent hyperpolarization ${ }^{2}$. On the other hand, in CA3 immediately continuing from $\mathrm{CA} 4$ as well as in CA4, all field potentials, interictal and elicited alike, exhibited slow positivity without exception. This is probably because in this area the apical dendrites are not uniform in direction ${ }^{24)}$ and they do not necessarily form a layer clearly separable from the cell bodies, and therefore the positivities due to the two kinds of hyperpolarizations cannot be distinguished from each other.

While a large spontaneous hyperpolarization occurs in the pyramidal cell of the kindled hippocampus, it never takes place in the normal hippocampus'). In the latter, a large hyperpolarization appears only when pyramidal cells are synchronously activated through electrical stimulation. In 
the former, however, a large hyperpolarization, comparable in size to that induced by supramaximal fornix stimulation, can appear spontaneously. The present investigation has demonstrated the existence of large population spikes at the onset or the early part of the interictal event. This, together with the existence of single action potentials preceding interictal hyperpolarizations ${ }^{1,2)}$, indicates that synchronous discharge of pyramidal cells is spontaneously taking place in the kindled hippocampus and therefore many recurrent inhibitory circuits are activated synchronously, resulting in the large interictal hyperpolarization. Thus it appears that spontaneously occurring synchronous activation of recurrent inhibitory circuits is one of the essential features of the kindled hippocampus. It should be noted that the interictal hyperpolarization never exceeded in magnitude the supramaximal fornix-evoked hyperpolarization. It is therefore evident that the powerful inhibition in the kindled hippocampus is brought about not through the enhancement of inhibition in the individual inhibitory circuits, but through the synchronous activation of numerous inhibitory circuits.

The author expresses his gratitude to Miss M. Hamada for preparing the photographic prints and other works, and to the members of the department for their cooperation in the experiments.

\section{References}

1) Fujita, Y. and Sakuranaga, M.: Spontaneous hyperpolarization in pyramidal cells of chronically stimulated rabbit hippocampus. Jpn. J. Physiol., 31, 879 889, 1981.

2) Fujita, Y., Harada, H., Takeuchi, T., Sato, H. and Minami, S.: Enhancement of EEG spikes and hyperpolarizations of pyramidal cells in the kindled hippocampus of the rabbit. Jpn. J. Physiol., 33, 227 238, 1983.

3) Ayala, G.F., Dichter, M., Gumnit, R.J., Matsumoto, H. and Spencer, W.A.: Genesis of epileptic interictal spikes; New knowledge of cortical feedback systems suggest a neurophysiological explanation of brief paroxysms. Brain Res., 52 , $1 \sim 17,1973$.

4) Dichter, M. and Spencer, W.A.: Penicillin-induced interictal discharges from the cat hippocampus. I. Characteristics and topographical features. J. Neurophysiol., 32, 649 662, 1969.

5) Dichter, M. and Spencer, W.A.: Penicillin-induced interictal discharges from the cat hippocampus. II. Mechanism underlying origin and restriction. J. Neurophysiol., 32, 663 687, 1969.

6) Hotson, J.R. and Prince, D.A.: A calcium-activated hyperpolarization follows repetitive firing in hippocampal neurons. J. Neurophysiol., 43, 409 419, 1980.

7) Nicoll, R.A. and Alger, B.E.: Synaptic excitation may activate calcium dependent potassium conductance in hippocampal pyramidal cells. Science, 212, 957 959, 1981.

8) Schwartzkroin, P.A. and Prince, D.A.: Cellular and field potential properties of epileptogenic hippocampal slices. Brain Res., 147, 117 130, 1978.

9) Schwartzkroin, P.A. and Pedley, T.A.: Slow depolarizing potentials in "epileptic" neurons. Epilepsia, 20, 267 279, 1979.

10) Wong, R.L.S. and Prince, D.A.: Afterpotential generation in hippocampal pyramidal cells. J. Neurophysiol., 45, 86 97, 1981.

11) Takeuchi, T.: EEG spikes and thereto corresponding hyperpolarizations of pyramidal cells in the hippocampus of the normal rabbit. Nippon Ika Daigaku Zasshi, 50, 844 851, 1983.

12) Fujita, Y., Sato, H., Minami, S. and Tazawa, Y.: Spontaneously occurring synchronous activation of pyramidal cells in the kindled hippocampus. J. Physiol. Soc. Jpn., 46, 395, 1984.

13) Fujita, Y., Sato, H., Takeuchi, T. and Minami, S.: Median raphe- and contralateral hippocampus-elicited EEG spikes which correspond to hyperpolarizations of pyramidal cells in the kindled hippocampus of the rabbit. Brain Res., 278, 313 317, 1983.

14) Fujita, Y.: Evidence for the existence of inhibitory postsynaptic potentials in dendrites and their functional significance in hippocampal pyramidal cells of adult rabbits. Brain Res., 175, 59 69, 1979.

15) Fujita, Y. and Nakamura, Y.: Effect of fornical stimulation upon CA1 and CA2 apical dendrite of rabbit's hippocampus. Jpn. J. Physiol., 11, 357 -368, 1961.

16) Fujita, Y. and Sakata, H.: Electrophysiological properties of CA1 and CA2 apical dendrites of rabbit hippocampus. J. Neurophysiol., 25, 209 222, 1962.

17) Fujita, Y.: Synaptic activation of dentate granule cells and its effect upon hippocampal pyramidal cells in rabbit. “Physiologie de L'Hippocampe”, p. 47 69, CNRS, Paris, 1962. 
18) Azmitia, E.C. and Segal, M.: An autoradiographic analysis of the differential ascending projection of the dorsal and median raphe nuclei in the rat. J. Comp. Neurol., 179, 641 668, 1978.

19) Babillier, P., Seguin, S., Petitjean, F., Salvert, D., Touret, H. and Jouvet, M.: The raphe nuclei of the cat brain stem; A topographical atlas of their efferent projections as revealed by autoradiography. Brain Res., 113, 449 486, 1976.

20) Fujita, Y., Takeuchi, T., Harada, H. and Matsuzawa, I.: The deactivating system of EEGs. J. Physiol. Soc. Jpn., 44, 397, 1982.

21) Harada, H.: EEG spikes (deactivating effects) produced by electrical stimulation of the brain stem in rabbit hippocampus. Nippon Ika Daigaku Zasshi, 50, 868 875, 1983.

22) Minami, S.: Stimulation of the raphe nuclei produces EEG spikes in the hippocampus of the normal rabbit. Nippon Ika Daigaku Zasshi, 51, 557 567, 1984.

23) Segal, M.: Physiological and pharmacological evidence for a serotonergic projection to the hippocampus. Brain Res., 94, $115 \sim 131,1975$.

24) Lorente de No, R.: Studies on the structure of the cerebral cortex. II. Continuation of the study of the ammonic system. J. Psychol. Neurol., 46, 113 177, 1934. 